\title{
SIBRA: Scalable Internet Bandwidth Reservation Architecture
}

\author{
Cristina Basescu*, Raphael M. Reischuk*, Pawel Szalachowski*, Adrian Perrig*, \\ Yao Zhang ${ }^{\dagger}$, Hsu-Chun Hsiao ${ }^{\ddagger}$, Ayumu Kubota ${ }^{\S}$, Jumpei Urakawa ${ }^{\S}$ \\ ${ }^{*}$ ETH Zurich, Switzerland \\ ${ }^{\dagger}$ Beihang University, China \\ ${ }^{\ddagger}$ National Taiwan University, Taiwan \\ $\S$ KDDI R\&D Laboratories Inc., Japan
}

\begin{abstract}
This paper proposes a Scalable Internet Bandwidth Reservation Architecture (SIBRA) as a new approach against DDoS attacks, which, until now, continue to be a menace on today's Internet. SIBRA provides scalable inter-domain resource allocations and botnet-size independence, an important property to realize why previous defense approaches are insufficient. Botnetsize independence enables two end hosts to set up communication regardless of the size of distributed botnets in any Autonomous System in the Internet. SIBRA thus ends the arms race between DDoS attackers and defenders. Furthermore, SIBRA is based on purely stateless operations for reservation renewal, flow monitoring, and policing, resulting in highly efficient router operation, which is demonstrated with a full implementation. Finally, SIBRA supports Dynamic Interdomain Leased Lines (DILLs), offering new business opportunities for ISPs.
\end{abstract}

\section{INTRODUCTION}

A recent extensive discussion among network administrators on the NANOG mailing list [4] pointedly reflects the current state of DDoS attacks and the trickiness of suitable defenses: defenses typically perform traffic scrubbing in ISPs or in the cloud, but attacks often surpassing 20-40 Gbps overwhelm the upstream link bandwidth and cause congestion that traffic scrubbing cannot handle. As attacks of up to 400 Gbps have recently been observed [5], no vital solution seems to be on the horizon that can defend the network against such large-scale flooding attacks.

Quality of service (QoS) architectures at different granularities, such as IntServ [42] and DiffServ [20], fail to provide end-to-end traffic guarantees at Internet scale: with billions of flows through the network core, routers cannot handle the per-flow state required by IntServ, whereas the behavior of DiffServ's traffic classification across different domains cannot guarantee consistent end-to-end connectivity.

Network capabilities [7, 24, 30, 44, 46] are not effective against attacks such as Coremelt [38] that build on legitimate low-bandwidth flows to swamp core network links. FLoc [24] in particular considers bot-contaminated domains, but it is ineffective in case of dispersed botnets.

Fair resource reservation mechanisms (per source [29], per destination [46], per flow [12, 42, 44], per computation [32],

Permission to freely reproduce all or part of this paper for noncommercial purposes is granted provided that copies bear this notice and the full citation on the first page. Reproduction for commercial purposes is strictly prohibited without the prior written consent of the Internet Society, the first-named author (for reproduction of an entire paper only), and the author's employer if the paper was prepared within the scope of employment.

NDSS '16, 21-24 February 2016, San Diego, CA, USA

Copyright 2016 Internet Society, ISBN 1-891562-41-X

http://dx.doi.org/10.14722/ndss.2016.23132 and per class [20]) are necessary to resolve link-flooding attacks, but are not sufficient: none of them provides botnetsize independence, a critical property for viable DDoS defense.

Botnet-size independence is the property in which a legitimate flow's allocated bandwidth does not diminish below the minimum allocation when the number of bots (even in other ASes in the world) increases. Per-flow and per-computation resource allocation, for instance, will reduce their allocated bandwidth towards 0 when the number of bots that share the corresponding resources increases.

To illustrate the importance of botnet-size independence, we observe how previous systems suffer from the tragedy of the network-link commons, which refers to the problem that the allocation of a shared resource will diminish toward an infinitesimally small allocation when many entities have the incentive to increase their "fair share" 1 In particular, per-flow fair sharing allocations (including per-class categorization of flows) suffer from this fate, as each source has an incentive to increase its share by simply creating more flows. However, even when the fair sharing system is not abused, the resulting allocations are too small to be useful. To explain in more detail, denoting $N$ as the number of end hosts in the Internet, persource or per-destination schemes could ideally conduct fair sharing of $O(1 / N)$ based on all potential sources or destinations that traverse a given link. However, with increasing hop-count distance of the link from the source or to the destination, the number of potential sources or destinations that traverse that link increases exponentially. Per-flow reservation performs even more poorly, allocating a bandwidth slice of only $O\left(1 / M^{2}\right)$ in the case of a Coremelt attack [38 between $M$ bots, and only $O(1 / M * P)$ during a Crossfire attack [21] with $P$ destination servers that can be contacted. In the presence of billions of end hosts engaged in end-to-end communication, the allocated bandwidth becomes too small to be useful.

In this paper, we propose a Scalable Internet Bandwidth Reservation Architecture (SIBRA), a novel bandwidth allocation system that operates at Internet-scale and resolves the drawbacks of prior systems. In a nutshell, SIBRA provides interdomain bandwidth allocations, which enable construction of Dynamic Interdomain Leased Lines (DILLs), in turn enabling new ISP business models. SIBRA's bandwidth reservations guarantee a minimal amount of bandwidth to each pair of end hosts by limiting the possible paths in end-to-end communica-

${ }^{1}$ We use this term following Garrett Hardin's Tragedy of the Commons [17], which according to the author has no technical solution, but instead "requires a fundamental extension in morality". As we should not expect attackers to show any of the latter, we believe in a technical solution at least for the Internet! 
tion. An important property of SIBRA is its per-flow stateless operation for reservation renewal, monitoring, and policing, which results in scalable and efficient router operation. SIBRA is fully implemented; our evaluation demonstrates its effectiveness.

\section{Goals, Assumptions, AND the AdVERsARY}

The goal of this paper is to defend against link-flooding attacks, in which distributed attackers collude by sending traffic to each other (Coremelt [38]) or to publicly accessible servers (Crossfire [21]) in order to exhaust the bandwidth of targeted servers and Internet backbone links. In the case of Coremelt, the traffic volume might not be limited (e.g., by TCP congestion control) since all participating hosts are under adversarial control and can thus run any protocol. In the case of Crossfire, distributed attackers collude by sending traffic to legitimate hosts in order to cut off network connections to selected servers. We note that other known attacks constitute a combination of the two cases above.

Adversary model. We assume that ASes may be malicious and misbehave by sending large amounts of traffic (bandwidth requests and data packets). We furthermore assume any AS in the world can contain malicious end hosts (e.g., as parts of larger botnets). In particular, there is no constraint on the distribution of compromised end hosts. However, attacks launched by routers (located inside ASes) that intentionally modify, delay, or drop traffic (beyond the natural drop rate) are out of the scope of this paper.

Desired properties. Under the defined adversary model, we postulate the following properties a link-flooding-resilient bandwidth reservation mechanism should satisfy:

- Botnet-size independence. The minimum amount of guaranteed bandwidth per end host does not diminish with an increasing number of bots.

- Per-flow stateless operation. The mechanism's overhead on routers should be negligible. In particular, backbone routers should not require per-flow, per-source, or perdestination state in the fastpath, which could lead to state exhaustion attacks ${ }^{2}$ Our analysis of real packet traces on core links supports this property (Section VIII-B).

- Scalability. The costs and overhead of the system should scale to the size of the Internet, including management and setup, AS contracts, router and end host computation and memory, as well as communication bandwidth.

Network assumptions. To achieve the properties we seek, we assume (i) a network architecture that provides sourcecontrollable network paths, and (ii) hierarchical bandwidth decomposition.

Concerning the first assumption of source-controllable network paths, we assume that routing paths (i.e., sequences of AS hops) are selected from several options by bandwidthrequesting sources (who then negotiate bandwidth with the destination and the intermediate AS hops). There are multiple routing protocols that provide such features: Pathlet routing [15], NIRA [45], and SCION [9, 48], where the

${ }^{2} \mathrm{~A}$ router's fastpath handles packet processing and forwarding on the line card, and is thus performance-critical. Routing protocols, network management, and flow setup are handled by the slowpath, which typically executes on the main router CPU and is thus less performance-critical. source can specify a path in the packet headers, or I3 [36] and Platypus [33], where the source specifies a sequence of forwarding nodes. We note that this first assumption may be of independent interest for ISPs since they may financially benefit [23].

Our second assumption of bandwidth decomposition is satisfied through a concept of domain isolation. To this end, we leverage SCION's isolation concept [9, 48] by grouping ASes into independent Isolation Domains (ISDs), each with an isolated control plane. Figure 1 depicts an example of 4 ISDs. The two end hosts $S$ and $D$ in different ISDs are connected by stitching three types of path segments together: an up-segment from $S$ to its ISD core, a core-segment within the Internet core (from source ISD to destination ISD), and a down-segment from $D$ 's ISD core to end host $D$. The ISD core refers to a set of top-tier ASes, the core ASes, that manage the ISD (depicted with a dark background in Figure 1). Intuitively, the isolation property yields that ASes inside an ISD can establish paths with bandwidth guarantees to the ISD core independently of bandwidth reservations in other ISDs. The bandwidth reservations for paths across ISDs will then be based on the reservations inside the ISDs, but will be lowerand upper-bounded for each end host. In particular, malicious entities will not be able to congest the network.

Furthermore, we assume that each end-to-end flow from $S$ to $D$ can be assigned a unique, non-hijackable flow identifier [6, 18, 28]; that ASes locally allocate resources to their internal end hosts; and that network links can fail and exhibit natural packet loss, which could lead to dropped reservation requests or dropped data packets.

\section{SIBRA DESIGN}

This section describes the design of SIBRA, in particular bandwidth reservations and their enforcement. After a brief overview, we describe SIBRA's reservation types in detail.

\section{A. SIBRA overview}

A key insight of SIBRA is its hierarchical decomposition of the bandwidth allocation problem to make management and configuration scale to the size of the Internet. More specifically, SIBRA makes use of (1) core contracts: long-term contracts amongst the core ASes of large-scale isolation domains (ISDs), (2) steady contracts: intermediate-term contracts amongst ASes within an ISD, and (3) ephemeral contracts: short-term contracts for end-to-end communication that leverage the longterm and intermediate-term contracts.

Thanks to this three-layer decomposition, on the order of 100 large-scale ISDs (e.g., composed by sets of countries or groups of companies) can scalably establish long-term core paths with guaranteed bandwidth between each other (the double continuous lines in Figure 1). Within each ISD, providers sell bandwidth to their customers, and customers can establish intermediate-term reservations for specific intraISD paths, which we call steady paths (the dashed lines in Figure 1). Steady paths are mostly used for connection setup traffic, but can also be used for low-bandwidth data traffic. Finally, core and steady paths in conjunction enable the creation of short-term end-to-end reservations across ISDs, which we call ephemeral paths (the solid green lines in Figure 1). Ephemeral paths, in contrast to steady paths, are used for the transmission of high-throughput data traffic. 


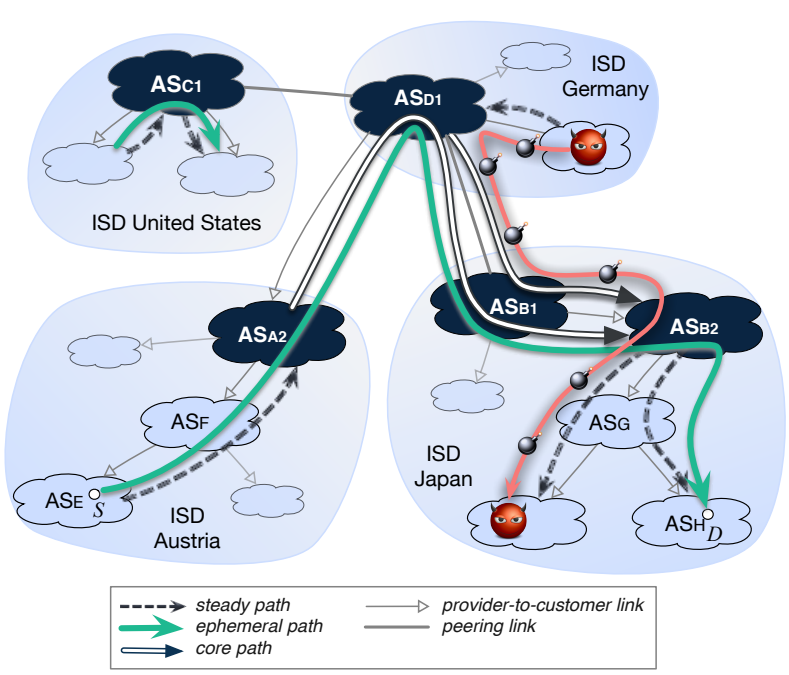

Fig. 1: Exemplary SIBRA topology with 4 isolation domains and their ASes (the core ASes are filled). The ephemeral path (green) from end host $S$ to end host $D$ is created along a steady up-path, a core path, and a steady down-path. The attack traffic (red) does not diminish the reserved bandwidth on ephemeral paths.

SIBRA paths are established over SIBRA links whose anatomy is depicted in Figure 2. $80 \%$ of the bandwidth of each SIBRA link is allocated for ephemeral traffic, $5 \%$ for steady traffic, and the remaining $15 \%$ for best-effort traffic. These proportions are flexible system parameters; we discuss the current choice in Section VIII-A. Note that the proportion for steady and ephemeral traffic constitutes an upper bound: in case the ephemeral bandwidth is not fully utilized, it is allocated to best-effort traffic (Section III-D).

An important feature of SIBRA is that steady paths, besides carrying the 5\% control traffic of links inside an ISD, also limit the bandwidth for ephemeral paths: An ephemeral path is created by launching a request through existing steady paths whose amounts of bandwidth determine - up to a fixed scaling factor - the bandwidth of the requested ephemeral path. More precisely, an ephemeral path is created through the combination of (i) a steady up-path in the source ISD, (ii) the steady part of a core path, and (iii) a steady down-path in the destination ISD ${ }^{3}$ The ephemeral path request uses only the steady portion of a link (the blue part in Figure 2); the actual ephemeral path traffic uses only the ephemeral portion of a link (the orange part in Figure 2). In other words, the more steady bandwidth a customer purchases locally within her ISD, the larger the fraction of ephemeral bandwidth she obtains to any other ISD in the Internet.

Based on these ideas, it becomes intuitively clear how botnet-size independence is achieved and how the tragedy of the network-link commons is resolved: Each pair of domains can obtain a minimum bandwidth allocation, based on their respective steady paths and based on the core contract. Thus, a botnet cannot influence the minimum allocation, no matter its size and distribution. A bot can only use up the bandwidth allocated to the AS it resides in, but not lower the minimum allocation of any other AS. It is thus in the responsibility of

${ }^{3}$ For instance, Figure 1 shows an ephemeral path from host $S$ in $A S_{E}$ to host $D$ in $A S_{H}$. If the source and destination are in the same ISD, then the core path may not be necessary, e.g., the ephemeral path inside the US ISD.

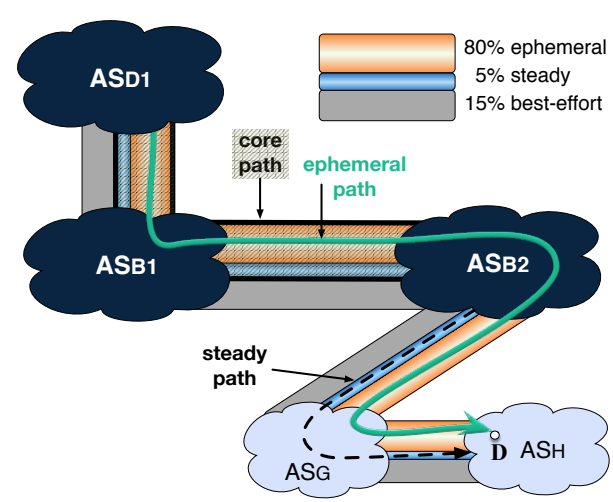

Fig. 2: The anatomy of SIBRA links: $80 \%$ of the link bandwidth is used for ephemeral traffic, 5\% for steady traffic, and 15\% for besteffort traffic. The core path from $A S_{D 1}$ to $A S_{B 2}$ comprises steady and ephemeral traffic, but excludes best-effort traffic.

an AS to manage its allocations, and thereby to prevent bots from obtaining resources of others within that AS.

In case an AS is dissatisfied with its minimum allocation, it can purchase more bandwidth for its steady paths, as well as request its core AS to purchase a larger allocation for the core contract, which the AS would likely need to pay for. An important point of these contracts is that, in order to scale, core contracts are purely neighbor-based: only neighboring ASes perform negotiations.

SIBRA's scalability is additionally based on a relatively low number of ephemeral paths, compared to all possible end-to-end paths in today's Internet, considered for instance by IntServ [42]. As mentioned above, an ephemeral path in SIBRA is fully determined by choosing two steady paths and a core path. The number of steady up-/down-paths an AS can simultaneously have is upper-bounded by a small SIBRA system parameter (e.g., 5 to 7), and the number of core paths is naturally upper-bounded by the number of ISDs.

To make SIBRA viable for practical applications, we need to ensure that all aspects of the system are scalable and efficient, which holds in particular for the frequent operations such as flow admission, reservation renewal, and monitoring and policing. For instance, all fastpath operations are per-flow stateless to avoid state-exhaustion attacks and to simplify the router architecture.

\section{B. Core paths}

Directly-connected core ASes (i.e., Tier-1 ISPs) are expected to agree on a guaranteed amount of bandwidth for traffic traversing their connecting links. We envision that ASes ratify such core contracts on mutual business advantages for their customers, on top of currently negotiated customer-to-provider or peering relations. Similar to SLAs, core contracts are long term (e.g., on the order of months) and can have an up-time associated (e.g., the bandwidth is guaranteed $99.99 \%$ of the time). Core contracts comprise steady and ephemeral traffic, as illustrated in the shaded part of Figure 2. If one of the ASes sends more traffic than agreed on, the AS is held accountable, according to the established contract.

Core contracts are initiated by receiver core ASes: each core AS observes the historical traffic values received on its neighboring links, and proposes in the core contracts similar values for the traffic the AS is willing to absorb. For instance, 


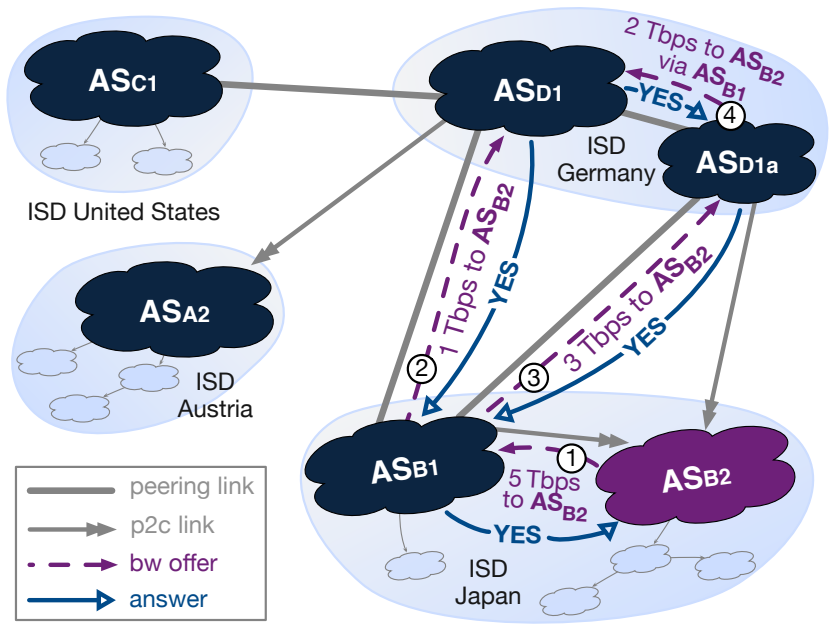

Fig. 3: Core contracts between core ASes $\left(A S_{D 1}, A S_{D 1 a}, A S_{B 1}, A S_{B 2}\right)$.

\begin{tabular}{clc}
\hline Destination & Path & Bandwidth \\
\hline \hline$A S_{B 2}$ & $A S_{B 1} \rightarrow A S_{B 2}$ & 1 Tbps \\
$A S_{B 2}$ & $A S_{D 1 a} \rightarrow A S_{B 1} \rightarrow A S_{B 2}$ & 2 Tbps \\
$\vdots$ & $\vdots$ & $\vdots$ \\
\hline
\end{tabular}

Fig. 4: Core contracts table at $A S_{D 1}$. Two core paths lead to $A S_{B 2}$.

in Figure 3, $A S_{B 2}$ proposes to absorb 5 Tbps of steady and ephemeral traffic from $A S_{B 1}$ (Step (1)), and $A S_{B 1}$ accepts. The contract is followed as long as $A S_{B 1}$ sends at most 5 Tbps to $A S_{B 2}$, regardless of whether $A S_{B 1}$ is the actual origin of the traffic, or $A S_{B 1}$ only forwards someone else's traffic to $A S_{B 2}$. For instance, $A S_{B 1}$ could forward traffic from $A S_{D 1}$ and $A S_{D 1 a}$ to $A S_{B 2}$. In the example, $A S_{B 1}$ offers to forward 1 Tbps from $A S_{D 1}$ (Step (2)), and 3 Tbps from $A S_{D 1 a}$ (Step (3). $A S_{D 1 a}$ extends the latter contract by proposing to $A S_{D 1}$ to absorb 2 Tbps towards $A S_{B 2}$ (Step (4)). After completion of the negotiation, $A S_{D 1}$ obtains guaranteed bandwidth to $A S_{B 2}$ along two core paths.

Figure 4 illustrates a local guaranteed-bandwidth table that stores such core paths for $A S_{D 1}$. The table resembles a forwarding table and may contain multiple entries for each destination core AS, one entry for each core path. It results from the contract proposals and the received acknowledgments for a specific destination, $A S_{B 2}$ in this case. For brevity's sake, Figure 4 shows only the entries for destination $A S_{B 2}$.

The bandwidth of a core path reflects the overall traffic volume exchanged between the source and the destination ASes. To bootstrap the process, each participating AS observes aggregate traffic volumes on its neighboring links, and initiates contracts with a bandwidth of $85 \%$ of the observed aggregate volume (5\% steady $+80 \%$ ephemeral). The initially estimated contracts are refined as dictated by the customer requirements and payments (explained below).

Scalability. The core contract proposals traverse only one link before being accepted or denied. For instance, in Figure 3 . $A S_{B 1}$ first accepts $A S_{B 2}$ 's proposal (Step (1)), and only afterwards, it submits its offers (Steps (2) and (3)). Achieving global consensus through immediate agreements is possible due to the destination-initiated process of establishing core contracts, in which the supported amount of traffic is already specified

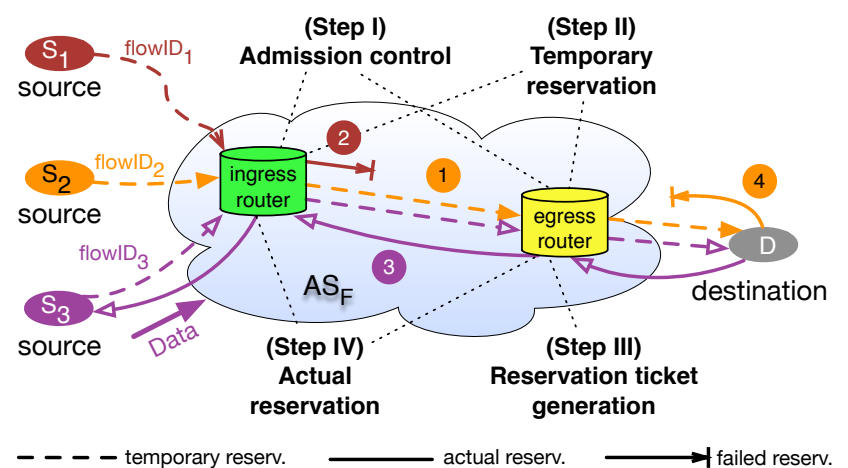

Fig. 5: Transit $A S_{F}$ processing reservation requests for sources $S_{1}$, $S_{2}, S_{3}$ and destination $D$

and can thus be decided based on local knowledge. In contrast, source-initiated requests would require a distributed consensus algorithm that would traverse all ASes whose agreement is required. SIBRA's design decision sacrifices such costly interactions for better scalability, achieving a core contract design that is scalable with the number of core ASes.

Payment. Core paths not only guarantee bandwidth between ISDs, they also regulate the traffic-related money flow between core ASes according to existing provider-to-customer (p2c) or peering (p2p) relationships (e.g., c2p between $A S_{B 2}$ and $A S_{B 1}$, and $\mathrm{p} 2 \mathrm{p}$ between $A S_{D 1}$ and $\left.A S_{B 1}\right)$.

Similar to today's state of affairs, we believe that market forces create a convergence of allocations and prices when ASes balance the bandwidth between their peers and adjust the contracts such that the direct core AS neighbors are satisfied. The neighbors, in turn, recursively adapt their contracts to satisfy the bandwidth requirements of their customers. Paying customers thus indirectly dictate to core ASes the destination ISDs of core paths and the specified bandwidth in the contracts.

\section{Steady paths}

Steady paths are intermediate-term, low-bandwidth reservations that are established by ASes for guaranteed communication availability within an ISD. We envision that the default validity of steady paths is on the order of minutes, but it can periodically be extended. An endpoint AS can voluntarily tear down its steady path before expiration and set up a new steady path. For example, in Figure 1. $A S_{E}$ sets up a steady path to $A S_{A 2}$, and $A S_{H}$ requests bandwidth guarantees from $A S_{B 2}$. As mentioned earlier, SIBRA uses steady paths for two purposes: (1) as communication guarantees for low-bandwidth traffic, and (2) as building block for ephemeral paths: to guarantee availability during connection setup and to perform weighted bandwidth reservations (Section III-D).

Reservation request. SIBRA leverages so-called SCION routing beacons [9] that disseminate top-down from the ISD core to the ASes. On their journey down, they collect AS-level path information as well as information about the current amount of available bandwidth (both steady and ephemeral) for each link. When a leaf AS receives such a routing beacon with information about a path segment, the AS can decide to submit

\footnotetext{
${ }^{4}$ We use the term destination in the following (and also in Figure 5 to stay as general as possible. For steady-path reservation requests, the destination is the ISD core; for ephemeral-path reservation requests, the destination will be another end host Section III-D).
} 
a reservation request that promotes the path segment to a steady path. In this case, the leaf AS (e.g., $A S_{E}$ in Figure 1. or $S_{3}$ in Figure 5) computes a new flow ID, chooses the amount of bandwidth and the expiration time, and sends a steady path reservation message up the path to the core. The requested amount of bandwidth can be chosen from a number of predefined bandwidth classes, introduced for monitoring optimization purposes (Section III-E).

Each intermediate AS on the path to the core performs admission control by verifying the availability of steady bandwidth to its neighbors on the path (Step I in Figure 5). Given the fact that inbound traffic from multiple ingress routers may converge at a single egress router, admission control is performed at both ingress and egress routers. Specifically, the ingress router of $A S_{i}$ checks the availability of steady bandwidth on the link $A S_{i-1} \rightarrow A S_{i}$, and the egress router of $A S_{i}$ on the link $A S_{i} \rightarrow A S_{i+1}$. If enough bandwidth is available at both the ingress and the egress router (Case $\mathbf{0}$ in Figure 5), both routers temporarily reserve the requested bandwidth (Step II). Subsequently, the egress router of $A S_{i}$ issues a cryptographically authenticated reservation token (RT) encoding the positive admission decision (Step III).

An RT generated by $A S_{i}$ is authenticated using a cryptographic key $K_{i}$ known only to $A S_{i}$, by which $A S_{i}$ can later verify if an RT embedded in the data packet is authentic. More specifically, the RT contains the authenticated ingress and the egress interfaces of $A S_{i}$, and the reservation request information. RTs are onion-authenticated to prevent an attacker from crafting a steady path from RT chunks:

$$
\begin{aligned}
& R T_{A S_{i}}=\text { ingress }_{A S_{i}} \| \text { egress }_{A S_{i}} \| \\
& \operatorname{MAC}_{K_{i}}\left(\text { ingress }_{A S_{i}} \| \text { egress }_{A S_{i}} \| \text { Request } \| R T_{A S_{i-1}}\right)
\end{aligned}
$$

where Request is defined as Bw req $\|$ ExpTime $\|$ flowID. We emphasize that steady path reservation flow identifiers are independent of TCP flow identifiers: A steady path can carry packets from multiple TCP flows, as long as these packets contain the RTs corresponding to the steady path in their header.

If at least one of the routers of $A S_{i}$ cannot meet the request (Case 2), it suggests an amount of bandwidth that could be offered instead, and adds this suggestion to the packet header. Although already failed, the request is still forwarded to the destination (i.e., to the ISD core in case of steady paths) to collect suggested amounts of bandwidth from subsequent ASes. This information helps the source make an informed and direct decision in a potential bandwidth re-negotiation.

As steady paths are only infrequently updated, scalability and efficiency of steady path updates are of secondary importance. However, $A S_{i}$ can still perform an efficient admission decision by simply considering the current utilization of its directly adjacent AS neighbors. Such an efficient mechanism is necessary for reservation requests (and renewals) to be fastpath operations, avoiding to access per-path state. In case of a positive admission decision, $A S_{i}$ needs to account for the steady path individually per leaf AS where the reservation originates from. Only slowpath operations, such as policing of misbehaving steady paths, need to access this per-path information about individual steady paths.

Confirmation and usage. When the reservation request reaches the destination $D$, the destination replies to the request- ing source (e.g., $S_{3}$ ) either by a confirmation message (Case 3 in Figure 5 containing the RTs accumulated in the request packet header, or by a rejection message (Case (4) containing the suggested bandwidth information collected before 4 As the confirmation message travels back to the source, every ingress and egress router accepts the reservation request and switches the reservation status from temporary to active (Step IV).

In order to use the reserved bandwidth for actual data traffic, the source includes the RTs in the packet header.

\section{Ephemeral paths}

Ephemeral paths are used for communication with guaranteed high bandwidth. Ephemeral paths are short-lived, only valid on the order of tens of seconds, and thus require continuous renewals through the life of the connection. The source, the destination, and any on-path AS can rapidly renegotiate the allocations. Figure 1 shows two ephemeral paths, one inside an ISD, one across three ISDs.

We emphasize that the amount of ephemeral bandwidth that is proportional to steady bandwidth may constitute a lower bound: If more ephemeral bandwidth is available (for instance since not everybody might be using his fair share of ephemeral bandwidth), requesters can choose a bandwidth class above the proportional ratio. In the spirit of fair allocation of joint resources, the lifetime of ephemeral paths is limited to 16 seconds in order to curtail the time of resource over-allocation. The details of the over-allocation, however, are out of scope and left for future work.

Ephemeral paths from steady paths. Ephemeral path requests bear many similarities with steady path requests, yet bootstrapping is different: An ephemeral path reservation is launched by an end host, as opposed to a steady path reservation that is launched by a leaf AS. The end host (e.g., host $S$ in Figure 1p first obtains a steady up-path starting at its AS (e.g., $A S_{E}$ ) to the ISD core, and a steady down-path starting at the destination ISD core (e.g., $A S_{B 2}$ ) to the destination leaf AS (e.g., $A S_{H}$ ). Joining these steady paths with an inter-ISD core path (e.g., from $A S_{A 2}$ to $A S_{B 2}$ ) results in an end-to-end path $P$, which is used to send the ephemeral path request from the source end host $S$ to the destination end host $D$ using allocated steady bandwidth.

More specifically, $S$ first generates a new flow ID, chooses an amount of bandwidth to request from SIBRA's predefined ephemeral bandwidth classes, and sends the ephemeral path request along path $P^{5}$ Recall that the path is composed of a steady up-path of $S$, a core path, and a steady down-path of $D$. The leaf AS where the source end host resides (e.g., $A S_{E}$ ) may decide to block the request in some cases, for instance if the bandwidth purchased by the leaf $\mathrm{AS}$ is insufficient. Each intermediate AS on path $P$ performs admission control through a weighted fair sharing mechanism that ensures the ephemeral bandwidth is directly proportional with its steady path bandwidth, as described next. The bandwidth reservation continues similarly to the steady path case.

If bots infest source and destination leaf ASes, these bots may try to exceed their fair share by requesting, respectively approving, excessively large amounts of bandwidth. To thwart

\footnotetext{
${ }^{5}$ Similarly to the steady path case, although an ephemeral path is identified by a flow ID, this flow ID is orthogonal to TCP flow IDs. A single ephemeral path can transport any data packets regardless of their layer-4 protocol.
} 
this attack, each leaf AS is responsible for splitting its purchased bandwidth among its end hosts according to its local policy, and for subsequently monitoring the usage.

Efficient weighted bandwidth fair sharing. The intuition behind SIBRA's weighted fair sharing for ephemeral bandwidth is that purchasing steady bandwidth (or generally spoken: bandwidth for control traffic) on a link $L$ guarantees a proportional amount of ephemeral bandwidth on $L$. In Figure 1 . the ephemeral bandwidth on the ephemeral path from end host $S$ to $D$ is proportional to the steady bandwidth on the steady up-path from $A S_{E}$ to core $A S_{A 2}$, and also proportional to the steady bandwidth on the steady down-path from core $A S_{B 2}$ down to $A S_{H}$. We explain the details of the three cases of intrasource-ISD links, core links, and intra-destination-ISD links in the following.

(1) Ephemeral bandwidth in the source ISD. For instance, a steady up-path of $500 \mathrm{kbps}$ traversing intra-ISD link $L$ guarantees $\frac{80}{5} \cdot 500 \mathrm{kbps}$ of ephemeral bandwidth on $L$. Note that $\frac{80}{5}=16$ is the ratio between ephemeral and steady bandwidth (Section III-A). Generally speaking, a steady up-path $S_{u}$ with steady bandwidth $s B W_{u}$ traversing $L$ can request ephemeral bandwidth of

$$
e B W_{u}=16 \cdot s B W_{u}
$$

Consequently, an AS that purchases a steady up-path $S_{u}$ can guarantee its customers a fixed amount of ephemeral bandwidth for customers' ephemeral path requests launched via $S_{u}$, regardless of the ephemeral path requests from other ASes on $L$.

To provide bandwidth guarantees on every link to a destination, SIBRA extends the influence of the purchased steady up-path bandwidth along the path to the destination AS. In fact, SIBRA's weighted fair sharing for ephemeral bandwidth on core paths includes the purchased steady up-path bandwidth, as explained in the following.

(2) Ephemeral bandwidth on core links. Let $s B W_{S}$ be the total amount of steady bandwidth sold by a core $A S_{S}$ for all steady paths in $A S_{S}$ 's ISD. Let $s B W_{u}$ be the reserved bandwidth sold for a particular steady up-path $S_{u}$ in this ISD. Let further $s B W_{C}$ be the control traffic bandwidth of a core path $C$ between the core ASes of the steady paths for $S$ and $D$. Then, ephemeral reservations on $C$ launched via $S_{u}$ can be up to

$$
e B W_{u C}=\frac{s B W_{u}}{s B W_{S}} \cdot 16 \cdot s B W_{C}
$$

In other words, the ephemeral bandwidth reservable on $C$ launched via steady path $S_{u}$ depends not only on the amount of total ephemeral bandwidth on $C$, but also on $S_{u}$ 's steady up-path bandwidth in relation to the total amount of steady up-path bandwidth purchased in $S_{u}$ 's ISD.

(3) Ephemeral bandwidth in the destination ISD. In the destination ISD, the weighted fair sharing is slightly more complex, but follows the ideas of the previous cases: the weighting includes the steady bandwidth of all steady uppaths and all steady down-paths, as well as the ratios of the bandwidth of the core contracts. Before explaining the details, we note that the reason for including also the steady downpaths is to give the destination AS control over the minimum amount of traffic it receives along ephemeral paths.
More precisely, an ephemeral path launched over steady up-path $S_{u}$ and steady down-path $S_{d}$ with core path $C$ in between obtains ephemeral bandwidth

$$
e B W_{u d}=\frac{C_{S \rightarrow D}}{C_{* \rightarrow D}} \cdot \frac{s B W_{u}}{s B W_{S}} \cdot 16 \cdot s B W_{d}
$$

where $C_{S \rightarrow D}$ is the bandwidth negotiated in the core contract for $C$ between the core ASes of $S$ and $D$, and $C_{* \rightarrow D}$ is the total amount of bandwidth negotiated in all core contracts between any core AS and D's core AS.

Equation 3 looks similar to Equation 2, with an additional factor in the weighting that reflects the ratio of incoming traffic from other core ASes. Intuitively, this factor assures that traffic from every other core AS obtains its fair share based on the bandwidth negotiated in the individual bilateral contracts.

Finally, the overall bandwidth for an ephemeral path between end hosts $S$ and $D$ launched over steady up-path $S_{u}$ reads

$$
e B W_{u C d}=\min \left(e B W_{u}, e B W_{u C}, e B W_{u d}\right)
$$

These equations compute the guaranteed bandwidth using the envisioned long-term ratio of $5 \%$ steady traffic, $80 \%$ ephemeral traffic, and $15 \%$ best-effort traffic. Ideally, the ratio should be adjustable by each AS, initially with an imbalance in favor of best-effort during incremental deployment of SIBRA, until the number of SIBRA subscribers increases. The overall bandwidth $e B W_{u C d}$ that can be obtained during early deployment is the minimum of the individual ratios for each AS and their link bandwidth. We discuss the choice of the ratio in Section VIII-A and its adaption in terms of an incremental deployment strategy in Section VI.

Fair sharing of steady paths. A challenging question is whether a fair sharing mechanism is necessary for steady bandwidth. A steady up-path is used solely by the AS that requested it, and its use is monitored by the AS, which splits the steady up-path bandwidth between its end hosts. In contrast, steady down-paths need to be revealed to several potential source ASes, either as private steady down-paths (e.g., for a company's internal services), or as public steady down-paths (e.g., for public services). To prevent a botnet residing in malicious source ASes from flooding steady downpaths, SIBRA uses a weighted fair sharing scheme similar to ephemeral paths: each AS using a steady down-path obtains a fair share proportional to its steady up-path, and its ISD's core path. We give the details of the scheme in Appendix A.

Efficient bandwidth usage via statistical multiplexing. Internet traffic often exhibits a cyclical pattern, with alternating levels of utilized bandwidth. In situations of low utilization, fixed allocations of bandwidth for steady and ephemeral paths that are unused would result in a waste of bandwidth. SIBRA reduces such bandwidth waste through statistical multiplexing, i.e., unused steady and ephemeral bandwidth is temporarily given to best-effort flows. A small slack of unallocated steady and ephemeral bandwidth still remains to accommodate new steady and ephemeral bandwidth requests. As more and more entities demand steady paths and their fair share of ephemeral paths, SIBRA gradually squeezes best-effort flows and releases the borrowed steady and ephemeral bandwidth up to the default allocations. 


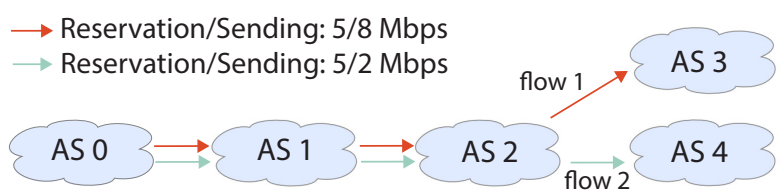

Fig. 6: Per-neighbor monitoring may label benign $A S_{1}$ malicious.

Renewal. End hosts can launch ephemeral path renewals to increase the reserved bandwidth and extend the expiration time of the ephemeral path. Since ephemeral reservations have a short lifetime, they are frequently renewed. Renewals are launched using the old reservation which contains the bandwidth class of the reservation; therefore routers can rapidly decide on the fastpath how much bandwidth they should allocate for the renewal, for instance if the bandwidth increased, decreased, or remained the same. Reservations are given a reservation index, incremented for each renewal of a specific ephemeral path. Reservations can be renewed anytime before they expire, and the end host is allowed to switch to the newer reservation at any time. However, the end host is not allowed to use both the old and the renewed reservation at the same time; Section III-F shows a mechanism to detect such misbehavior.

\section{E. Priority traffic monitoring and policing}

Flows that violate their reservations may undermine the guarantees of other legitimate flows. An ideal monitoring algorithm should immediately catch every such malicious flow. This, however, would be too expensive for line-rate traffic in the Internet core.

Instead, as the first line of defense, SIBRA relies on edge ASes to perform fine-grained traffic monitoring. Edge ASes rely on flow IDs to check each flow's bandwidth usage and compare it against the reserved bandwidth for that flow ID, which is stored by each AS locally during the reservation request. Previous research has shown that per-flow slowpath operations are feasible at the edge of the network [37].

Monitoring on transit ASes, however, needs to be processed on the fastpath. To detect misbehaving ASes that purposely fail to regulate their own traffic, SIBRA deploys a lightweight monitoring mechanism in transit ASes. First, each AS monitors the bandwidth usage of incoming traffic from each neighbor AS and compares it against the total bandwidth reserved for that neighbor. Such coarse-grained monitoring timely detects a misbehaving neighbor that failed to correctly police its traffic.

Why per-neighbor monitoring is insufficient. There are cases, though, when per-neighbor monitoring in transit ASes is insufficient. Figure 6 depicts two flows originating in $A S_{0}$, each having reserved $5 \mathrm{Mbps}$. Flow 1 is malicious and sends traffic with $8 \mathrm{Mbps}$, while flow 2 underuses its reservation. $A S_{0}$ hence does not properly monitor its flows. When $A S_{1}$ performs perneighbor monitoring, it can only notice that, in the aggregate, it receives $10 \mathrm{Mbps}$ from $A S_{0}$ and sends $10 \mathrm{Mbps}$ to $A S_{2}$. However, when the two flows diverge, $A S_{2}$ detects flow 1 as malicious and holds $A S_{1}$ responsible, although $A S_{1}$ properly performed per-neighbor monitoring.

For this reason, SIBRA additionally utilizes fine-grained probabilistic monitoring of individual flows at the transit ASes, using a a recently proposed technique [43]. Each transit AS monitors, per given time interval, all the flows in a number of randomly chosen bandwidth classes. Recall that the bandwidth class of a flow is authenticated by the RTs in the packet header. In case the average bandwidth utilization of a flow during that time interval exceeds the flow's bandwidth class, the flow is classified as malicious and added to a blacklist, preventing its renewal.

We emphasize that transit ASes perform monitoring on the fastpath. Only in case of suspicion of misbehavior, these ASes perform out-of-band slowpath monitoring to police misbehaving neighbors and flows.

To localize the origin AS of the malicious flow, an AS informs the previous AS of the misbehaving flow. In response, the previous AS can simply monitor that specific flow explicitly. If the violation persists, the suspicious previous-hop neighbor is likely to be malicious. Then, the AS can punish it, for instance, by terminating their contract.

\section{F. Flow renewal monitoring and policing}

A successful ephemeral path renewal replaces the old reservation, therefore the renewal receives the same flow ID as the old reservation. However, SIBRA paths allow for RTs with overlapping validity periods. Therefore, if multiple renewals occur before the ephemeral path expires, the source would be in possession of multiple sets of valid RTs: Some corresponding to the ephemeral path with the previous bandwidth class and old expiration time, and the others corresponding to the new values for bandwidth class and expiration time, along the same path. Since all sets of RTs are associated with the same flow ID, routers would overwrite their per-flow entries with the new bandwidth class.

A malicious end host could thus exploit renewals by using both sets of RTs, old and new, during the overlap time of the RTs, thus using more bandwidth than the amount reserved. To prevent such misuse, end hosts are not allowed to use old RTs after having used the renewed RTs. When renewals use the same bandwidth class as the old reservation, simultaneous use of old and new RTs is detected by the per-class monitoring mechanism (as described above) since the usage is jointly accounted under the same flow ID.

We now consider the case when the renewed bandwidth class is different from the old one. The edge AS performs perflow stateful inspection and is supposed to filter out traffic that violates the sending rule. Therefore, the edge AS can be held accountable by other ASes for improperly filtering traffic. In transit ASes, however, we propose a probabilistic approach for catching this type of misbehavior. ASes maintain one Bloom filter [10] per currently active expiration time and per bandwidth class. Since an RT is maximally valid for 16 seconds and the time granularity is 4 seconds, 4 Bloom filters are needed per bandwidth class, to record flow IDs that use the bandwidth class within that time period. We will discuss the details about time discretization in SIBRA in Section IV-A For an incoming packet with a reservation in a monitored class $C$, ASes simply store the tuple $\langle$ flow ID, reservation index $\rangle$ in the Bloom filter of class $C$. By probabilistically inspecting some of these Bloom filters, each AS notices whether a flow ID uses two different bandwidth classes during a time period.

We further optimize the monitoring algorithm as follows. SIBRA selects a small number of classes to monitor at a given moment in time, therefore ASes store Bloom filters only for the few monitored traffic classes. In addition, SIBRA does 
not investigate all Bloom filters: We observe that, when the renewed bandwidth is much higher or much lower than the previous bandwidth, using both the old and new reservations would incur an insignificant bandwidth overuse. Therefore, if a certain reservation index is used in class $C$, SIBRA investigates only the Bloom filters of the classes whose bandwidth values are comparable to $C$ 's bandwidth (the comparability of classes is discussed in Section IV-A. SIBRA investigates whether in these Bloom filters an index reservation index $+i$ is present, where $i \in\{0,1, \ldots, 15\}$ chosen randomly ( $i=0$ detects whether the end host maliciously reuses the same reservation index). If found, ASes increment a violation counter for the source of that flow ID. The violation counter allows for Bloom filter false positives. When the violation counter exceeds a threshold, an alarm is raised for that sender. Therefore, the more packets an attacker sends, the higher the probability of detection. The policing push back technique can then localize the source AS of the misbehaving flow.

\section{G. Dealing with failures}

While bandwidth guarantees along fixed network paths allow for a scalable design, link failures can still disrupt these paths and thus render the reservations futile. In fact, leaf ASes and end hosts are rather interested in obtaining a bandwidth guarantee than obtaining a specific network path for their traffic.

SIBRA deals with link failures using two mechanisms: (1) a failure detection technique to remove reservations along faulty paths, and (2) a failure tolerance technique to provide guarantees in the presence of failures. For (1), SIBRA uses short expiration times for reservations and keep-alive mechanisms. Steady paths expire within 3 minutes of creation, but leaf ASes can extend the steady paths' lifetime using keepalive messages. Ephemeral paths have a default lifetime of 16 seconds, which can be extended by source end hosts through renewals. Unless keep-alive messages or renewals are used, reservations are removed from the system within their default expiration time. By construction, a new reservation cannot be created on top of faulty paths. For (2), SIBRA allows leaf ASes to register multiple disjoint steady paths. We also envision source end hosts being able to choose a bandwidth reservation service with high reliability, which would use a small number of disjoint ephemeral paths to the same destination.

\section{H. Dynamic Interdomain Leased Lines}

Businesses use leased lines to achieve highly reliable communication links. ISPs implement leased lines virtually through reserved resources on existing networks, or physically through dedicated network links. Leased lines are very costly, can require weeks to be set up, and are challenging to establish across several ISPs.

A natural desire is to achieve properties similar to traditional leased lines, but more efficiently. GEANT offers a service called "Bandwidth-On-Demand" (BoD), which is implemented through the InterDomain Controller Protocol [1] to perform resource allocations across the participating providers [14]. Although BoD is a promising step, the allocations are still heavy-weight and require per-flow state.

With SIBRA's properties, ISPs can offer lightweight Dynamic Interdomain Leased Lines (DILLs). A DILL can be composed by two longer-lived steady paths, connected through a core path, or dynamically set up with an ephemeral path that is constantly renewed. Thanks to the lightweight operation of SIBRA, DILLs can be set up with an RTT setup message and are immediately usable. Our discussions with operators of availability-critical services have shown that the DILL model has sparked high interest among operators.

To enable long-term DILLs, valid on the order of weeks, the concept of ephemeral paths in SIBRA could be reframed: long-term DILLs could use the same techniques for monitoring and policing as ephemeral paths, however, they would also introduce new challenges. To enable long-term DILLs, ISPs need to ensure bandwidth availability even when DILLs are not actively used, as opposed to ephemeral bandwidth, which can be temporarily used by best-effort flows. For this purpose, ISPs could allocate a percentage of their link bandwidth for DILLs, besides steady, ephemeral, and best-effort paths. Additionally, for availability in the face of link failures, ISPs would need to consider active failover mechanisms. For instance, in architectures that provide path choice, ISPs could leverage disjoint multipath reservations concentrated in a highly available DILL. A detailed design though is out of scope for this paper.

\section{IMPLEMENTATION}

We present the implementation of senders and routers to launch a reservation request and to use a reservation. We rely on efficient data structures and algorithms that enable fastpath processing in the common case and explain the infrequent operations when SIBRA needs slowpath processing.

\section{A. Bandwidth reservation setup}

Sender implementation. A reservation request initiator specifies the following configuration parameters: a flow ID (128 bits), a reservation expiration time (16 bits), bandwidth classes for forward and/or reverse directions (5 bits each), a path direction type ( 2 bits), and a reservation index (4 bits). SIBRA considers time at a granularity of 4 seconds (which we call SIBRA seconds). By default, steady paths thus have an initial lifetime of 45 SIBRA seconds, and ephemeral paths of 4 SIBRA seconds; nevertheless, these paths can be renewed at any time. All reservations start at the request time.

We chose SIBRA's bandwidth classes to cover a meaningful range for steady and ephemeral traffic: there are 12 steady bandwidth classes according to the formula $16 \cdot \sqrt{2^{i}} \mathrm{kbps}$, where $i \in\{0,1, \ldots, 11\}$, ranging from $16 \mathrm{kbps}$ to $\sim 724 \mathrm{kbps}$; and 20 ephemeral bandwidth classes according to the formula $256 \cdot \sqrt{2^{i}} \mathrm{kbps}$, where $i \in\{0,1, \ldots, 19\}$, ranging from $256 \mathrm{kbps}$ to $\sim 185 \mathrm{Mbps}$. The exponential growth allows for a fine-grained allocation of smaller bandwidth values, but more coarse-grained allocation of larger bandwidth values. Additionally, it enables efficient monitoring of flow renewals, with a small number of classes having comparable bandwidth.

The path direction type is a flag that indicates, for a $\langle$ requester, destination $\rangle$ pair, either a uni-directional reservation, for traffic either sent or received by the requester; or bi-directional, for traffic sent and received by the requester. The reservation index is a number specific to a flow, incremented every time the reservation corresponding to the flow is renewed.

Bandwidth reservation and accounting. To efficiently manage and account for bandwidth reservations, SIBRA routers 
maintain the following data structures: (1) a bandwidth table, i.e., an array of size $k$ storing the currently reserved bandwidth for each of the router's $k$ neighbors; (2) an accounting table, i.e., a table with tuples containing the flow ID of a reservation, the expiration time, the bandwidth class, and the neighbor to/from whom the reservation is specified; (3) a pending table, i.e., a table (of similar structure as the accounting table) that stores pending reservations. A reservation is said to be pending if it has been requested, but not used for data transmission. A reservation with flow ID $i$ is said to be active when data has been transmitted using $i$, i.e., the router has seen $i$ in a data packet. A reservation for $i$ is said to be expired if the router has not seen packets containing $i$ within a time frame of $\ell$ SIBRA seconds (details below).

To decide whether a requested amount $b w_{r}$ can be reserved, routers perform admission control by comparing $b w_{r}$ with the entry in the bandwidth table for the specified neighbor 6 In case sufficient bandwidth is available, the request's flow ID, the expiration time, the request's bandwidth class, and the neighbor are added to the pending table. The requested amount $b w_{r}$ is also added to the respective entry in the bandwidth table. Yet, at this point, the router does not add information about the request to the accounting table. The reason is that the request may fail at a later point, in which case the accounting table update would have to be reverted. In a periodic background process, the router checks whether there are entries in the pending table older than 300 milliseconds (sufficient to allow for an Internet round trip time $\overline{7}^{7}$ ). Such entries are considered failed reservations, and thus they are deleted from the pending table and the corresponding reserved bandwidth is freed and updated in the bandwidth table.

If the router sees a data packet with flow ID $i$ for the first time, it implies that the reservation for flow ID $i$ was accepted by all routers on the path. The reservation becomes active and the entry with flow ID $i$ is then removed from the pending table and added to the accounting table.

To periodically reclaim unused ephemeral bandwidth of expired reservations, a router periodically removes the amount of expired bandwidth from the bandwidth table. The expiration parameter $\ell$ (e.g., $1 \leq \ell \leq 5$ ) specifies the lifetime (in SIBRA seconds) of pending reservations. In order to keep reservations active (even if no data is transmitted), a source simply sends a keep-alive message within $\ell$ SIBRA seconds. In a periodic background process, the router then iterates over the accounting table's entries that correspond to the last $\ell$ SIBRA seconds. More specifically, the router checks whether the listed flow IDs occur in a Bloom filter that is filled while forwarding data packets: to enable fastpath operation, the flow ID of each incoming data packet is stored in a Bloom filter, not in the accounting table. Bandwidth reclaim is then processed in the slowpath.

Intermediate AS implementation. The MAC operation of RTs are implemented using CBC-MAC based on AES. Our AES implementation uses AESni [16], a fast instruction set available on Intel and AMD CPUs, which requires only 4.15

\footnotetext{
${ }^{6}$ The reason for considering only the current amount of available bandwidth when making the admission decision is justified by the monotonicity of reservations: reservations can never be set up to start in the future, hence, in the next SIBRA second, there cannot be less bandwidth available than in the current SIBRA second (unless new reservations are requested).

${ }^{7}$ http://www.caida.org/research/performance/rtt/walrus 0202
}

cycles per byte to encrypt a $1 \mathrm{kB}$ buffer in $\mathrm{CBC}$ mode. The key necessary for the MAC operation is expanded once at the AS and then used for all RTs generated by that AS. SIBRA uses 32 bits for MACs, which constitutes an optimization, yet provides sufficient security: a forgery will be detected with probability $1-2^{-32}$.

During a reservation request, the header for the positive admission of a flow contains the request configuration values set by the sender and the list of RTs generated so far. A field Hops is used to locate the correct offset for a newly generated RT. In addition, a field Extension Flag indicates the request path type (bi-/uni-directional), the request status (successful or failed), and whether the packet carries a reservation request or a reservation confirmation.

When a request does not pass the admission control, then the corresponding router sets the extension flag to failed, marks its own AS in the Decline AS* field, and resets Hops to zero. Starting with this AS, every subsequent AS on the path towards destination adds a Bandwidth Offer field with the offered bandwidth.

We implemented SIBRA on top of a SCION-enabled network, which provides path control. Our SIBRA implementation provides end-host support through a SIBRA-enabled gateway, which contains modules for reservation requests and their confirmation, SCION encapsulation, decapsulation, and a traffic hijacking module. The last element is implemented via NetFilter Queue [41], and it allows to tunnel legacy IP traffic to a remote host through the SIBRA-enabled SCION network. Such a design provides SIBRA's benefits to legacy software, as well as facilitates SIBRA's deployment.

The SIBRA packet header contains SCION-relevant information, such as src/dst addresses, forwarding path as opaque fields (OFs), the current OF/RT indicator, and an optional extension field in which SIBRA's reservation request messages are encoded. We implemented SIBRA in SCION using extension headers.

\section{Evaluation}

\section{A. Processing on router}

We first evaluated SIBRA with respect to the processing overhead on routers. For our evaluation, we used a traffic generator that initiated bandwidth reservation requests, and sent traffic within existing reservations. The traffic generator was connected to a software router that performed admission control of the request packets, RT verification, monitoring for the existent reservations, and then forwarded the packets. Every experiment was conducted 1000 times. We considered routers placed in both edge and core ASes, however processing time only differed for monitoring operations. All the tests were conducted on a PC with an Intel Intel Xeon E5-2680 $2.7 \mathrm{GHz}$ and 16 GB of RAM, running Linux (64-bit).

First, we investigated the time required by a router to process the SIBRA reservation request. The average time to process a reservation request was $9.1 \mu \mathrm{s}$, resulting in about 109890 that can be processed per second.

Then, we tested the speed of the data packet processing. To this end, we used our high-performance implementation that deploys Intel's DPDK framework for networking operations,

${ }^{8}$ http://dpdk.org/ 
and the AESni extension for cryptographic operations. We set the packet length to 1500 bytes. We measured the time of SIBRA processing (i.e., packet parsing and RT verification). It took $0.040 \mu \mathrm{s}$ on average to process a single packet, thus a router is capable to process about 25 million data packets per second. (Note that these times do not include interactions with the NIC).

Next, we investigated the performance of monitoring in the core for two scenarios: 1 and 100 attackers. The average processing time was $11.24 \mu \mathrm{s}$ for a single attacker, and $9.91 \mu \mathrm{s}$ for 100 attackers. As the results show, the average processing time decreases with an increasing number of attackers, as blacklisted flows are processed faster.

\section{B. Bandwidth guarantees under botnet attacks}

To show SIBRA's resilience to Denial of Capability (DoC) and Coremelt attacks, we run a simulation on an Internet-scale topology. In our simulation, the attackers attempt to exhaust the bandwidth of the links common with legitimate flows. We compare our results with TVA [46], Portcullis [32], and STRIDE [19], obtained using the same configuration.

Method. Our Internet-scale topology is based on a CAIDA dataset [2] that contains 49752 ASes and the links among them as observed from today's Internet. Based on these connections, we grouped the ASes into five ISDs, representing five continent-based regions. For our simulation we chose the two biggest ISDs: $I S D_{1}$ containing 21619 , and $I S D_{2}$ containing 6039 ASes. The core of each ISD is formed by Tier-1 ISPs. We set the capacity of the core link between $I S D_{1}$ and $I S D_{2}$ to $40 \mathrm{Gbps}$. Inside each ISD, we set the capacity of core links to $10 \mathrm{Gbps}$, the capacity of links between a core AS and a Tier-2 AS to $2.4 \mathrm{Gbps}$, and all other links to $640 \mathrm{Mbps}$. Steady paths and core paths were established before the experiment.

In both attack scenarios, the attackers (compromised hosts) are distributed uniformly at random in different ASes. Legitimate sources reside in two ASes (i.e., each AS contains 100 legitimate sources). We further use the same parameters as the related work: a 5\% rate limit for reservation requests, and request packets of 125 bytes. All the sources (including attackers) send 10 requests per second. According to Mirkovic et al. [27], we set 4 seconds as the request timeout.

DoC Attack. We simulate both intra-ISD and inter-ISD DoC attacks. For the intra-ISD case, source and destination ASes are within $I S D_{2}$, and $I S D_{2}$ contains 1000 contaminated ASes. All the requests, from benign and malicious ASes, traverse the same link in the core. In the inter-ISD scenario, the source resides in $I S D_{1}$ and the destination resides in $I S D_{2}$, there are 500 contaminated ASes in each ISD, and all the requests traverse the same links in the core.

Figures 7(a) and 7(b) show the fraction of successfully delivered capability requests (success ratio) correlated to the number of active attackers. For both cases (intra- and inter-ISD DoC attacks), TVA and Portcullis perform similarly: on core links, legitimate requests mingle with malicious ones. Afterwards, since the link bandwidth decreases after traversing the core, there is a rapid increase in the request packets' queueing time. Consequently, the success ratio decreases. TVA's success ratio stabilizes around $40 \%$. Portcullis uses computational puzzles, and the request packets with a higher computational level are forwarded first. Hence, when more attackers with optimal strategy [32] appear, the time to compute a puzzle increases accordingly, leading to a decrease of the success ratio to 0 when the computation time exceeds 4 seconds. In STRIDE, the ISD core has no protection, but traffic inside $I S D_{2}$ has a higher priority than traffic coming from $I S D_{1}$. Thus, during the intra-ISD attack, STRIDE's success ratio stays $100 \%$ until the core becomes congested. However, in the inter-ISD case, STRIDE's performance declines dramatically, since a majority of requests from $I S D_{1}$ are dropped if any core link in $I S D_{2}$ is congested. SIBRA successfully delivers all the legitimate requests, in both attack scenarios, because SIBRA requests are launched using steady paths, and steady paths guarantee a fair share of control traffic along core paths.

Coremelt Attack. We simulate a Coremelt attack with the following settings: $I S D_{2}$ contains 500 pairs of contaminated ASes (selected uniformly at random), which communicate using ephemeral paths, each with a throughput of $8 \mathrm{kbps}$ of their $256 \mathrm{kbps}$ reservations. The source and the destination also communicate using an ephemeral path, of $800 \mathrm{kbps}$. All the ephemeral paths in the experiment traverse the same core link. We measure the bandwidth obtained when the source sends to the destination a $1 \mathrm{MB}$ file.

Figure 7(c) shows that the congestion on the core link degrades the file transfer time in STRIDE to over 100 seconds. TVA, which uses per-destination queues to forward authorized traffic, performs slightly worse than Portcullis, simulated using per-source weighted fair sharing based on the computational level. SIBRA outperforms the other schemes, because it gives a lower bound on the bandwidth obtained for the file transfer, due to its weighted fair sharing based on the steady paths.

\section{Lower bound on bandwidth fair share}

We simulate the bandwidth obtained by new ephemeral paths when requests for ephemeral paths arrive from both benign and malicious sources. We considered a scenario where all the requests are forwarded using the same steady down-path (SIBRA's worst case for weighted fair sharing).

The legitimate steady up-path from the source AS carried 5 requests per second, and has a bandwidth of $362 \mathrm{kbps}$. There were approximately 50 attackers on every malicious up-path, and each attacker sent one request per second. The attackers' steady up-path bandwidth was randomly selected from our steady bandwidth classes (16 kbps to $724 \mathrm{kbps}$ ). The bandwidth requested for ephemeral paths ranged from $256 \mathrm{kbps}$ up to $11.6 \mathrm{Mbps}$.

The result for this setting is presented in Figure 8 (a). The green line shows the real-time reservable bandwidth, that changes dynamically but finally stabilizes around $2.5 \mathrm{Mbps}$. At time interval 100, the number of attackers and steady uppaths used for requesting ephemeral paths increases. However, SIBRA guarantees that reservable bandwidth remains stable despite the increasing numbers of attackers. This is due to the fair share, which is not affected by the number of attackers with steady paths.

\section{Reservation request loss tolerance}

Next, we simulate the influence of packet loss on ephemeral bandwidth reservation. We assume that at every second there are 1000 reservation requests sent, with the following parameters: variable path length (5-10), random bandwidth 


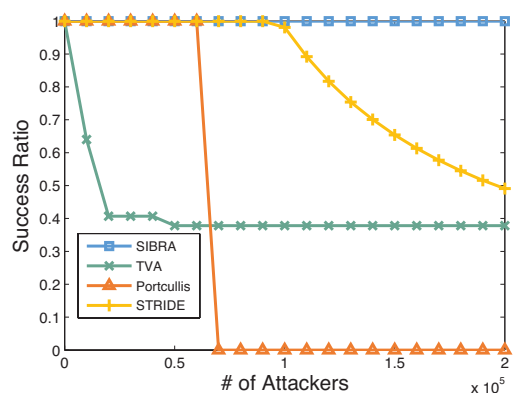

(a)

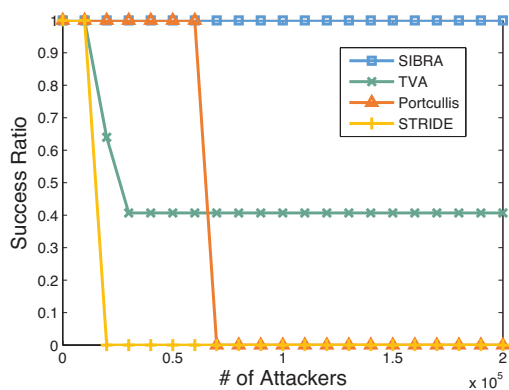

(b)

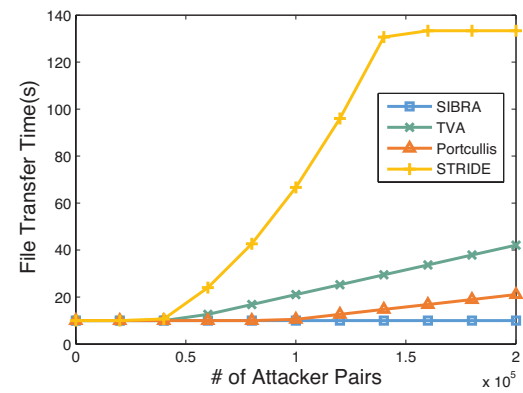

(c)

Fig. 7: Comparative simulation results for TVA, Portcullis, STRIDE, and SIBRA against Intra-ISD DoC attack 7(a)] Inter-ISD DoC attack 7(b) and Coremelt attack $7(c)$

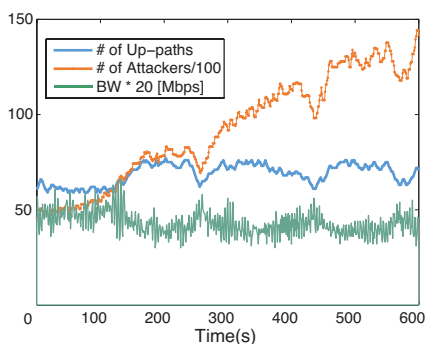

(a)

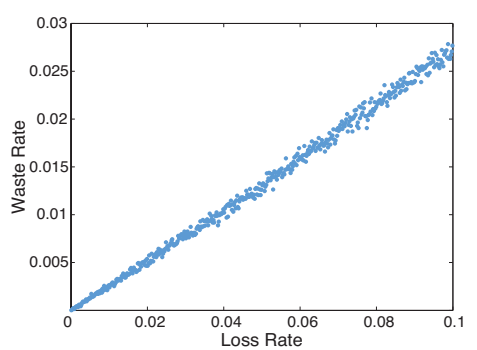

(b)
Fig. 8: Simulation results on SIBRA's availability. (a) shows the existence of the reservable bound for bandwidth requests. Note that the bandwidth (green line) in the figure is multiplied by 20 for improved readability. (b) presents the resilience of bandwidth reservation against packet loss.

(50 kbps - 6.4 Mbps), variable packet loss rate (0-10\%), and RTT set to 1 second. Similar to Portcullis [32] and TVA [46], we assume that request packets are limited to $5 \%$ of the entire link capacity.

In our simulation, we consider packet loss for both reservation request and reply packets. This setting introduced unused bandwidth reservation on the routers that had already processed the packet, until bandwidth reclaim occurs. We express the bandwidth waste rate $r_{\text {waste }}$ as unused reserved bandwidth divided by the sum of reserved bandwidth.

As shown in Figure $8(\mathrm{~b})$, even at a loss rate of $5 \%$, the corresponding $r_{\text {waste }}$ is no more than $1.4 \%$. Moreover, the diagram indicates that $r_{\text {waste }}$ increases linearly when the loss rate rises, which shows that SIBRA tolerates packet loss well, thus providing robust bandwidth reservation.

\section{INCREMENTAL DEPLOYMENT}

Within a single ISP network, deployment of SIBRA does not require major changes in the underlying infrastructure since the ISP can utilize its existing core network with protocolindependent transport like MPLS. The ISP can thus build a "SIBRA-ready" network by adding new customer/provider edge routers and setting up MPLS tunnels with reserved bandwidth among them to traverse the traditional network fabric. A global-scale inter-ISP deployment is more challenging, because a simple overlay approach with IP-tunneling would not provide the contiguous bandwidth reservation required for SIBRA. To take full advantage of SIBRA, ISPs need direct links to interconnect their SIBRA routers. Therefore, in its initial deployment phase, we envision a SIBRA network operated by a small group of ISPs with mutual connectivity.

An essential question is whether such a partially-deployed new network infrastructure provides immediate financial benefits for early adopters, and subsequently attracts new ISPs. The business example of the startup company Aryaka is similar to SIBRA regarding the deployment purposes. Aryaka has successfully established a private core network infrastructure, dedicated to optimize WAN traffic between Aryaka's Points of Presence (POPs) across the world. These POPs deploy Aryaka's proprietary WAN optimization protocols, and enterprise customers' distributed business sites located near POPs benefit from application acceleration. By offering a global network solution, Aryaka gained the interest of regional ISPs that want to provide WAN optimization beyond their own regions. Aryaka is continuously expanding its edge infrastructure through Tier-3 and Tier-4 ISPs. Yet, as opposed to SIBRA, by using a private core network, Aryaka's solution comes at a high cost, and may be even more costly to scale to all ASes in the Internet.

Similar to the case of Aryaka, we expect SIBRA's deployment to begin at the core, between a few Tier-1 ISPs that seek to provide DILLs spanning their joint regions. These early adopters may quickly monetize the SIBRA bandwidth reservation service by selling DILLs to their direct customers. Gradually, the SIBRA network would expand through new ISP collaborators interested in providing bandwidth reservation beyond their own regions. ISPs have the incentive to support SIBRA, as they can draw traffic towards them, and also appeal to both existing and new clients who desire effective DDoS protection, thus increasing the ISPs' revenues.

During the expansion of SIBRA, ISPs are likely to start SIBRA deployment with lower ratios for steady and ephemeral bandwidth, suitable for the needs of a small number of initial SIBRA customers. Meanwhile, best-effort customers still enjoy a throughput similar to that before SIBRA deployment. As the number of SIBRA subscribers increases, ISPs could locally adjust the ratios towards an increased steady and ephemeral proportion, and persuade their providers to follow, as well as adjust their core contracts accordingly. As more and more customers shift from best-effort to SIBRA, best-effort traffic obtains a smaller ratio. Depending on their customer segmentation, ISPs could either adjust best-effort subscriptions to the new network traffic, or increase their link capacity.

We evaluated a potential deployment plan for SIBRA 


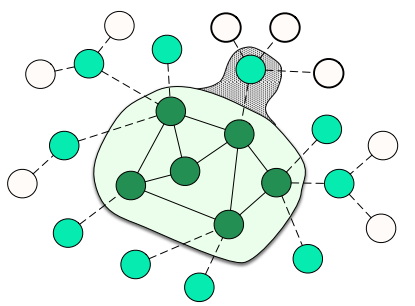

Fig. 9: Deploying ISPs (dark colors) gain revenue from all their neighbors (medium colors) potentially buying guaranteed bandwidth. The deploying region extends through neighbors (patterned area), with their direct neighbors as potential buyers (bold outline).

using the AS topology from CAIDA9 in the following setting. We considered a set of initial adopters, tier-1 ISPs selected uniformly at random. Potential adopters in the next deployment round are the neighbors of the deploying nodes, as depicted in Figure 9, such that there is always a contiguous region of deploying ASes. We consider rational potential adopters, which deploy SIBRA only if they can monetize the guaranteedbandwidth service by selling it to their neighbors. Such neighbors would buy the service if the traffic they originate can use DILLs up to their destinations. Thus, we compare the traffic originating at a buyer neighbor AS that can use DILLs, compared to the total amount of traffic originating at the same neighbor AS. Since traffic information between ASes is usually confidential, we approximate the traffic using a model introduced by Chan et al. [11]: the traffic between a source and a destination AS is represented by the product of the ASes' IP spaces. We obtained the data on the AS-IP-space mapping from CAIDA 10

When the set of initial deployers consists of three ASes, next round adopters could monetize SIBRA on a percentage of traffic between $40 \%-48 \%$. Four initial adopters lead to potential SIBRA traffic of $47 \%-49 \%$, and five initial adopters to $50 \%-52 \%$. We conclude that deployment starting at the Internet core greatly leverages the incremental deployment of SIBRA.

\section{USE CASES}

With the flexible lifetime of DILLs, ranging from tens of seconds to weeks on-demand, SIBRA brings immediate benefits to applications where guaranteed availability matters. These applications comprise critical infrastructures, such as financial services and smart electric grids, as well as business applications, such as videoconferencing and reliable data sharing in health care. As discussed above, setting up leased lines in these cases may take several weeks and may become prohibitively expensive: it is costly to install leased lines between each pair of domains, and also to connect each domain through a leased line to a central location in order to build up a star topology.

Critical infrastructures. Financial services, for instance transaction processing from payment terminals, would become more reliable when using SIBRA DILLs: since DILLs guarantee availability even in the presence of adversarial traffic, payment requests and their confirmations would always obtain a guaranteed minimum bandwidth. DILLs could also be used for remote monitoring of power grids: a minimum guaran-

\footnotetext{
"http://www.caida.org/data/as-relationships/

10 http://data.caida.org/datasets/routing/routeviews-prefix2as/
}

teed bandwidth would be suitable to deliver the monitored parameters, independent of malicious hosts exchanging traffic. Telemedicine is another use case of practical relevance: the technology uses telecommunication to provide remote health care - often in critical cases or emergency situations where interruptions could have fatal consequences.

Business-critical applications. Videoconferencing between the remote sites of a company receives increasing importance as a convenient way to foster collaborations while reducing travel costs. Short-lived and easily installable DILLs provide the necessary guaranteed on-demand bandwidth for reliably exchanging video traffic. Another application is reliable ondemand sharing of biomedical data for big-data processing, complementing the efforts of improving health care quality and cost in initiatives such as Big Data to Knowledge launched by the US National Institutes of Health (NIH) [26].

\section{DISCUSSION}

\section{A. On the choice of bandwidth proportions for SIBRA links}

Recall that in Section III-A we assigned $80 \%, 15 \%$, and 5\% of a link's bandwidth to ephemeral, best-effort, and steady paths, respectively. This parameter choice is justified through an analysis of today's actual Internet traffic.

- First to notice is that the majority of traffic constitutes persistent high-bandwidth connections: for example in Australia, we see that Netflix's video connections contribute to more than $50 \%$ of the entire Internet traffic [3]. Given an additional amount of traffic from other large video providers such as Youtube and Facebook, we estimate ephemeral paths to require roughly $70-90 \%$ of a link's bandwidth.

- Best-effort is still important for some types of lowbandwidth connections: email, news, and SSH traffic could continue as best-effort traffic, totaling $3.69 \%$ of the Internet traffic [22]; similar the case for DNS traffic totaling $0.17 \%$ of the Internet traffic [22]. In addition, very short-lived flows (that is flows with a lifetime less than $256 \mathrm{~ms}$ ) with very few packets (the median flow contains 37 packets [39]) are unlikely to establish SIBRA reservations, simply to avoid the round-trip time of the reservation setup. Such flows sum up to $5.6 \%$ of the Internet traffic [39] and can thus also be categorized under best-effort.

- Finally, regarding the amount of bandwidth for steady paths and connection-establishment traffic, we conducted an experiment using the inter-AS traffic summary by a DDoS detection system at one of the largest tier-1 ISPs. With a 10-day recording of this data, we found that only $0.5 \%$ of the $1.724 \times 10^{13}$ packets were connection establishment packets. To enable communication guarantees for low-bandwidth traffic, including bandwidth reservation request packets, we designed SIBRA to allocate tenfold of the amount measured.

Since it is hard to specify the actual bandwidth proportions precisely, we use $80 \%, 15 \%$, and $5 \%$ as initial values and note that these values can be re-adjusted at any point in the future.

We recall from Section III-D that, in addition to the parameter choice, SIBRA's statistical multiplexing between the traffic classes helps to dynamically balance the traffic. We expect that in particular the long-lived reservations are not 


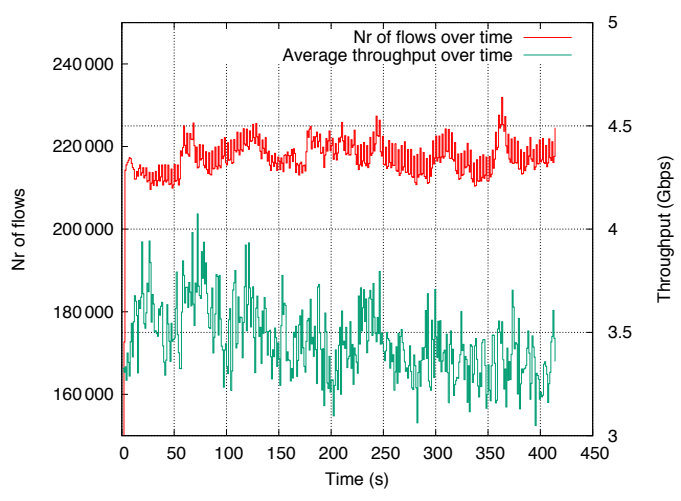

Fig. 10: The number of active flows every second and their throughput, observed on a 10 Gbps Internet core link.

always fully utilized, in which case best-effort traffic can be transmitted instead.

\section{B. Per-flow stateless operations are necessary}

To understand the amount of per-flow storage state required on the fastpath, we investigate the number of active flows per second as seen by a core router in today's Internet. We used anonymized one-hour Internet traces from CAIDA, collected in July 2014. The traces contain all the packets that traversed a 10 Gbps Internet core link of a Tier-1 ISP in the United States, between San Jose and Los Angeles.

Figure 10 depicts our findings as the number of active flows on the core link at a granularity of one second, for a total duration of 412 seconds. We observe that the number of flows varies around 220000 , with a boundary effect at the beginning of the data set. These flows sum into a throughput between 3 and 4 Gbps - a link load of $30 \%$ to $40 \%$. A large core router switching 1 Tbps (with 100 such 10 Gbps links) would thus observe $22 \times 10^{6}$ flows per second in the normal case, considering a link load of only $40 \%$. In an attack case, adversaries could greatly inflate the number of flows by launching connections between bots, as in Coremelt [38]. Schuchard et al. already analyzed attacks that can exhaust the router memory [34]. All these results suggest storing per-flow state in the fastpath, on the line card, becomes prohibitively expensive, even more so when the core link load increases.

\section{Case study: achievable ephemeral bandwidth on core links}

A central point of SIBRA is to guarantee a sufficient amount of bandwidth using today's infrastructure, even for reservations that span multiple ISDs. A central question is how much bandwidth an end-domain could minimally obtain if globally all domains attempt to obtain their maximum fair share. To investigate this point, we considered a scenario with Australia as destination, and all non-Australian leaf ASes in the world reserving ephemeral bandwidth to Australia. We picked Australia because with its 24 million inhabitants, it represents a major economy, and it already experienced infrastructure congestion in today's Internet [3]. While its geographical location hinders laying new cables, Australia is well-suited for our study aiming to determine a lower bound on the amount of bandwidth SIBRA core links can expect. Other countries, especially those situated on larger continents, typically feature higher-bandwidth connectivity, as laying cables on land is easier than in the ocean.

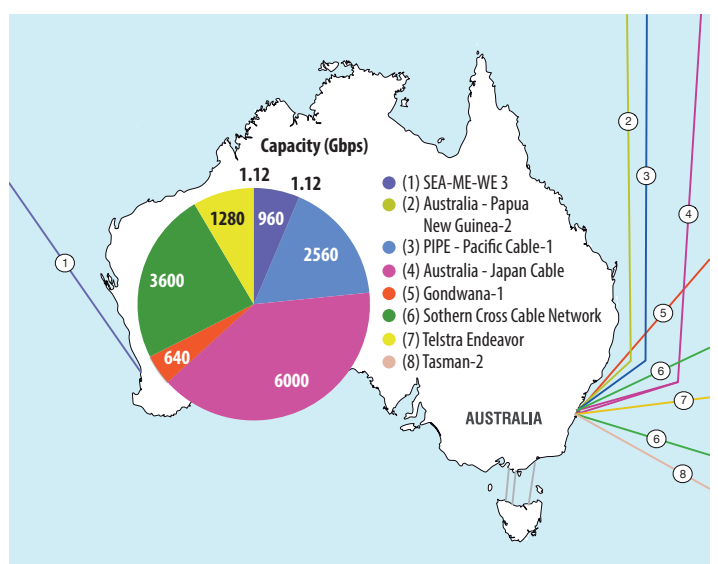

Fig. 11: Australia submarine link map, including link capacities.

Figure 11 illustrates the current submarine link map of Australia, including the name and capacity of the links ${ }^{11}$ The entire traffic traverses these links. For simplicity, we assume guaranteed bandwidth is split equally between leaf ASes. In practice, however, the bandwidth is proportional to the size of the steady paths of the leaf ASes (Section IIII). We considered two cases: (i) the worst case, i.e., when all reservations are squeezed over the same link - in our case, we chose the highest-bandwidth cable, namely the Australia-Japan Cable (6 Tbps), and (ii) the best case, i.e., when the reservations are distributed across all cables (totaling $15.04 \mathrm{Tbps}$ ). In contrast to other architectures, SIBRA's underlying architecture, SCION, enables the use of multi-path communication for the traffic between a source and a destination, along several core links.

We have determined the number of leaf ASes in the world, using the AS topology from CAIDA ${ }^{9}$, and counted 32428 nonAustralian leaf ASes using the AS number and location 12 After the analysis, we found that each non-Australian leaf AS obtains a fair share of (i) $185.02 \mathrm{Mbps}$ (148 Mbps for ephemeral traffic), or (ii) $463.86 \mathrm{Mbps}$ (371.08 Mbps for ephemeral traffic). We thus conclude that SIBRA's fair sharing scheme offers a substantial amount of bandwidth through an efficient use of the current Internet infrastructure. In case this amount is insufficient, an AS could purchase additional bandwidth for a specific destination from its core AS.

The prospects are even brighter: considering the planned undersea physical infrastructure development, the capacity of the cables connecting Australia with the rest of the world would increase by $168 \mathrm{Tbps}$ by the beginning of 2018. With such an increase, the fair share on SIBRA's core links becomes 5.64 Gbps per leaf AS in case (ii).

\section{RELATED WORK}

Capability-based mechanisms [7, 19, 24, 30, 32, 44, 46] aim at isolating legitimate flows from malicious DDoS attack traffic. Network capabilities are access tokens issued by onpath entities (e.g., routers and destination) to the source. Only packets carrying such network capabilities are allowed to use a privileged channel. Capability-based schemes, how-

\footnotetext{
${ }^{11} \mathrm{http}: / /$ www.submarinecablemap.com/ illustrates the submarine link map. The link capacities were obtained from various resources, e.g., the AustraliaJapan Cable capacity from http://www.ajcable.com/company-history/

${ }^{12} \mathrm{http}: / /$ data.caida.org/datasets/as-organizations/
} 
ever, require additional defense mechanisms against Denial of Capability attacks [8] and against attacks with colluding hosts or legitimate-looking bots [21, 38]. To address DoC attacks, TVA [46] tags each packet with a path identifier which is based on the ingress interface of the traversing ASes. The path identifier is used to perform fair queueing of the request packets at the routers. However, sources residing further away from the congested link will suffer a significant disadvantage. Portcullis [32] deploys computational puzzles to provide per-computation fair sharing of the request channel. Such proof-of-work schemes, however, are too expensive to protect every data packet. Moreover, Portcullis does not provide the property of botnet-size independence. Floc [24] fair-shares link bandwidth of individual flows and differentiates between legitimate and attack flows for a given link. However, such coarse-grained per-AS fair sharing may not always be effective; in particular, low-rate attack flows can often not be precisely differentiated. CoDef [25] is a collaborative defense mechanism in which a congested AS asks the source ASes to limit their bandwidth to a specific upper bound and to use a specific path. Source ASes that continue sending flows that exceed their requested quota are classified as malicious. CoDef does not prevent congestion in the first place, but instead retroactively handles one congested link at a time. Since congestion can still occur on links, sources cannot be given a guarantee for reaching a destination. STRIDE [19] is a capability-based DDoS protection architecture that builds on several concepts from SCION [9, 48]. Although STRIDE shares similarities with SIBRA (steady paths and ephemeral paths), STRIDE lacks intra-core and inter-ISD communication guarantees; STRIDE's intra-domain guarantees are built on the assumption of congestion-free core networks. Moreover, STRIDE lacks monitoring and policing mechanisms, as well as an implementation.

Resource allocation. Several queuing protocols [31, 35, 37] have been proposed to approximate fair bandwidth allocation at routers. Their correctness, however, relies on the trustworthiness of the routers and flow identifiers. The Path Computation Element (PCE) architecture [13, 40] computes inter-AS routes and enables resource allocation across AS boundaries in Generalized Multi-Protocol Label Switching (GMPLS) Traffic Engineered networks. However, the discovery of inter-AS PCE path fragments discloses information about other cooperating AS, such as the internal topology. Some ASes will be reluctant to share this information due to confidentiality reasons.

Resource reservation. RSVP [47] is a signaling protocol for bandwidth reservation. Because RSVP is not designed with security in mind, the reservation may fail due to DDoS attacks. RSVP requires the sender (e.g., a host or an AS when RSVP aggregation is used as specified in RFC 3175) to make an end-to-end reservation to the receiver(s), causing a quadratic number of control messages (in the number of entities) in the network and quadratic state on the intermediate routers.

\section{COnclusions}

Through hierarchical decomposition of resource reservations, SIBRA is the first scalable architecture that provides interdomain bandwidth guarantees - achieving botnet-size independence and resolving even sophisticated DDoS attacks such as Coremelt [38] and Crossfire [21]. SIBRA ends the arms race between DDoS attackers and defenders, as it provides guaranteed resource reservations regardless of the attacker's botnet size. A salient property of SIBRA is that it can be built without requiring per-flow state in the fastpath of a router, resulting in a simple router design and high-speed packet processing. We anticipate that SIBRA becomes a game changer in the battle against large-scale DDoS attacks.

\section{ACKNOWLEDGMENTS}

We would like to thank Virgil Gligor, Chris Pappas, Christian Rossow, Stephen Shirley, and Laurent Vanbever for insightful discussions and their valuable comments throughout the evolution of this project. We also thank Xiaoyou Wang, Dominik Roos, and Takayuki Sasaki for their help with the implementation and evaluation of SIBRA.

The research leading to these results has received funding from the European Research Council under the European Union's Seventh Framework Programme (FP7/2007-2013) / ERC grant agreement 617605 . We also gratefully acknowledge support by ETH Zurich, and NSF under award number CNS1040801. The research was also supported by a gift from KDDI.

\section{REFERENCES}

[1] "Inter-domain controller (IDC) protocol specification," http://www. controlplane.net/idcp-v1.1-ns/idc-protocol-specification-v1.1.pdf 2010

[2] "Center for Applied Internet Data Analysis (CAIDA)," http://www. caida.org/home/ 2014

[3] "Netflix congesting the Australian Internet," http://www.smh.com.au/ digital-life/digital-life-news/these-graphs-show-the-impact-netflix-ishaving-on-the-australian-internet-20150402-1mdc1i.html 2015.

[4] "North American Network Operators' Group," https://www.nanog.org/ list 2015.

[5] "Technical Details Behind a 400Gbps NTP Amplification DDoS Attack," https://blog.cloudflare.com/technical-details-behind-a-400gbpsntp-amplification-ddos-attack 2015.

[6] D. G. Andersen, H. Balakrishnan, N. Feamster, T. Koponen, D. Moon, and S. Shenker, "Accountable Internet Protocol (AIP)," in ACM SIGCOMM, 2008.

[7] T. Anderson, T. Roscoe, and D. Wetherall, "Preventing Internet Denialof-Service with Capabilities," ACM SIGCOMM Computer Communication Review, 2004

[8] K. Argyraki and D. R. Cheriton, "Network Capabilities: The Good, the Bad and the Ugly," in ACM HotNets, 2005.

[9] D. Barrera, R. M. Reischuk, P. Szalachowski, and A. Perrig, "SCION five years later: Revisiting scalability, control, and isolation on nextgeneration networks," arXiv e-prints, 2015.

[10] B. H. Bloom, "Space/time trade-offs in hash coding with allowable errors," Communications of the ACM, 1970.

[11] H. Chan, D. Dash, A. Perrig, and H. Zhang, "Modeling adoptability of secure BGP protocol," in ACM SIGCOMM, 2006.

[12] A. Demers, S. Keshav, and S. Shenker, "Analysis and simulation of a fair queueing algorithm," ACM SIGCOMM Comp. Comm. Rev., 1989.

[13] A. Farrel, J.-P. Vasseur, and J. Ash, "A path computation element (PCE)based architecture," Tech. Rep., 2006.

[14] GEANT, "Bandwidth on demand," http://geant3.archive.geant.net/ service/BoD/pages/home.aspx 2015.

[15] P. Godfrey, I. Ganichev, S. Shenker, and I. Stoica, "Pathlet routing," in ACM SIGCOMM Comp. Comm. Rev., 2009.

[16] S. Gueron, "Intel Advanced Encryption Standard (AES) New Instructions Set," Intel, 2010, white paper 323641-001, Revision 3.

[17] G. Hardin, "The tragedy of the commons," Science, 1968.

[18] T. Heer and S. Varjonen, "Host identity protocol certificates," 2011. 
[19] H.-C. Hsiao, T. H.-J. Kim, S. B. Lee, X. Zhang, S. Yoo, V. Gligor, and A. Perrig, "STRIDE: Sanctuary trail - refuge from internet DDoS entrapment," in AsiaCCS, 2013.

[20] F. B. J. Babiarz, K. Chan, "Configuration Guidelines for DiffServ Service Classes."

[21] M. S. Kang, S. B. Lee, and V. D. Gligor, "The Crossfire Attack," in IEEE $S \& P, 2013$.

[22] C. Labovitz, S. Iekel-Johnson, D. McPherson, J. Oberheide, and F. Jahanian, "Internet Inter-Domain Traffic," ACM SIGCOMM, 2010.

[23] P. Laskowski, B. Johnson, and J. Chuang, "User-directed routing: From theory, towards practice," in ACM NetEcon, 2008.

[24] S. B. Lee and V. D. Gligor, "FLoc: Dependable link access for legitimate traffic in flooding attacks," in IEEE ICDCS, 2010.

[25] S. B. Lee, M. S. Kang, and V. D. Gligor, "CoDef: Collaborative defense against large-scale link-flooding attacks," in ACM CoNEXT, 2013.

[26] R. Margolis, L. Derr, M. Dunn, M. Huerta, J. Larkin, J. Sheehan, M. Guyer, and E. D. Green, "The National Institutes of Health's Big Data to Knowledge (BD2K) initiative: capitalizing on biomedical big data," Journal of the American Medical Informatics Association, 2014.

[27] J. Mirkovic, S. Fahmy, P. Reiher, and R. K. Thomas, "How to test DoS defenses," in $C A T C H, 2009$.

[28] R. Moskowitz, P. Jokela, T. R. Henderson, and T. Heer, "Host identity protocol version 2."

[29] J. Nagle, "On Packet Switches with Infinite Storage," 1985.

[30] M. Natu and J. Mirkovic, "Fine-grained capabilities for flooding DDoS defense using client reputations," in ACM LSAD, 2007.

[31] R. Pan, B. Prabhakar, and K. Psounis, "CHOKe-a stateless active queue management scheme for approximating fair bandwidth allocation," in IEEE INFOCOM, 2000.

[32] B. Parno, D. Wendlandt, E. Shi, A. Perrig, B. Maggs, and Y.-C. Hu, "Portcullis: Protecting Connection Setup from Denial-of-Capability Attacks," in ACM SIGCOMM, 2007.

[33] B. Raghavan, P. Verkaik, and A. C. Snoeren, "Secure and policycompliant source routing," IEEE/ACM Transactions on Networking, vol. 17, no. 3, 2009.

[34] M. Schuchard, A. Mohaisen, D. Foo Kune, N. Hopper, Y. Kim, and E. Y. Vasserman, "Losing control of the internet: using the data plane to attack the control plane," in ACM CCS, 2010.

[35] M. Shreedhar and G. Varghese, "Efficient fair queuing using deficit round-robin," IEEE/ACM Transactions on Networking, 1996.

[36] I. Stoica, D. Adkins, S. Zhuang, S. Shenker, and S. Surana, "Internet Indirection Infrastructure," ACM SIGCOMM Comp. Comm. Rev., 2002.

[37] I. Stoica, S. Shenker, and H. Zhang, "Core-Stateless Fair Queueing: A Scalable Architecture to Approximate Fair Bandwidth Allocations in High-Speed Networks," IEEE/ACM Transactions on Networking, 2003.

[38] A. Studer and A. Perrig, "The Coremelt attack," in ESORICS, 2009.

[39] B. Trammell and D. Schatzmann, "On Flow Concurrency in the Internet and its Implications for Capacity Sharing," in ACM CSWS, 2012.

[40] J. Vasseur and J. Le Roux, "Path computation element communication protocol," IETF RFC 5557, 2009.

[41] H. Welte and P. N. Ayuso, "The netfilter.org libnetfilter_queue project," http://www.netfilter.org/projects/libnetfilter_queue//. 2014.

[42] J. Wroclawski, "The Use of RSVP with IETF Integrated Services," 1997.

[43] H. Wu, H.-C. Hsiao, and Y.-C. Hu, "Efficient large flow detection over arbitrary windows: An algorithm exact outside an ambiguity region," in ACM IMC, 2014

[44] A. Yaar, A. Perrig, and D. Song, "SIFF: A Stateless Internet Flow Filter to Mitigate DDoS Flooding Attacks," in IEEE S\&P, 2004.

[45] X. Yang, D. Clark, and A. W. Berger, "Nira: A new inter-domain routing architecture," IEEE/ACM Transactions on Networking, 2007.

[46] X. Yang, D. Wetherall, and T. Anderson, "A DoS-limiting network architecture," ACM SIGCOMM Comp. Comm. Rev., 2005.

[47] L. Zhang, S. Deering, D. Estrin, S. Shenker, and D. Zappala, "RSVP: A New Resource ReSerVation Protocol," IEEE Network, 1993.
[48] X. Zhang, H.-C. Hsiao, G. Hasker, H. Chan, A. Perrig, and D. G. Andersen, "SCION: Scalability, Control, and Isolation on Next-generation Networks," in IEEE $S \& P, 2011$. 


\section{APPENDIX}

\section{A. Fair sharing of steady down-paths}

Recall from Section III-B that core ASes negotiate core contracts to set up core paths among each other (the double continuous lines in Figure 1). The reserved bandwidth for those core paths is negotiated based on aggregated traffic volumes as observed in the past. The question we consider in the following is how the reserved bandwidth is split among the customers of the core ASes. More precisely, we describe a sharing mechanism that assigns each leaf AS $E$ a fair amount of bandwidth for $E$ 's traffic traversing the core paths. Intuitively, fair in this context means proportional to the amount of bandwidth that $E$ has purchased for its steady up-paths to the core AS. In contrast to the fair sharing mechanism for ephemeral paths (Section III-D), the equations we introduce here do not require the additional weighting factor $16=\frac{80}{5}$ given by the ratio of ephemeral and steady bandwidth.

Steady bandwidth on core links. The steady bandwidth of a core path $\mathbf{C}=\left\langle A S_{C 1}, \ldots, A S_{C n}\right\rangle$ between core $A S_{C 1}$ and a destination core $A S_{C n}$ is split between all customer ASes of $A S_{C 1}$, weighted with the bandwidth of the steady up-path each customer AS uses.

Let $s B W_{u *}$ be the total amount of steady bandwidth sold by a core $A S_{C 1}$ for all its steady paths. Let $s B W_{u}$ be the reserved bandwidth sold by $A S_{C 1}$ for a particular steady up-path $S_{u}$. Let further $s B W_{\mathbf{C}}$ be the steady bandwidth of core path $\mathbf{C}$. Then the steady traffic on $\mathbf{C}$ launched via $S_{u}$ can be up to:

$$
s B W_{u C}=\frac{s B W_{u}}{s B W_{u *}} \cdot s B W_{\mathbf{C}}
$$

Steady bandwidth in the destination ISD. In the destination ISD, the weighted fair sharing follows the same intuition, this time consisting first of a fair sharing mechanism between different ISDs, then between the ASes in the same ISD.

More precisely, steady traffic launched over steady up-path $S_{u}$ and steady down-path $S_{d}$, with core path $\mathbf{C}$ in between, obtains a throughput:

$$
s B W_{u C d}=\frac{C_{C 1 \rightarrow C n}}{C_{* \rightarrow C n}} \cdot \frac{s B W_{u}}{s B W_{u *}} \cdot s B W_{d}
$$

where $C_{C 1 \rightarrow C n}$ is the bandwidth negotiated in C's core contract between core $A S_{C 1}$ (source ISD) and core $A S_{C n}$ (destination ISD), $C_{* \rightarrow C n}$ is the total amount of bandwidth negotiated in the core contracts between any core AS and the destination ISD's core $A S_{C n}$, and $s B W_{d}$ is the reserved bandwidth sold by core $A S_{C n}$ for steady down-path $d$.

The second case is that of local steady traffic in the destination ISD, which does not traverse any core path. In fact, this happens only when the steady up- and down-paths cross at the same core AS, otherwise traffic would traverse a core path between the core ASes of the destination ISD (see Figure 1.

This case introduces a preference $\rho \in(0,1)$ that splits the bandwidth of the steady down-path between traffic that traverses core paths, and traffic that does not.
Let $s B W_{u *}$ be the total bandwidth of all steady up-paths to a given core AS in the destination ISD. Then, a particular steady up-path among them, say with bandwidth $s B W_{u}$, obtains steady bandwidth on the down-path $d$ of

$$
s B W_{u d}^{\rho}=\frac{s B W_{u}}{s B W_{u *}} \cdot \rho \cdot s B W_{d}
$$

Traffic from external ISDs is weighted accordingly, extending Equation 6.

$$
s B W_{u C d}^{\rho}=(1-\rho) \cdot s B W_{u C d}
$$

Authentication of bandwidth values. To compute $s B W_{u C}$ and $s B W_{u C d}$, ASes use the bandwidth values for steady up-paths and core paths included in the SIBRA packet headers. In order to prevent a malicious AS from increasing its fair share by tampering with the bandwidth values, SIBRA requires the core ASes of each ISD to sign the bandwidth of steady up-paths inside the ISD, as well as the bandwidth of core paths starting at the ISD core.

Signing can take place when the steady paths are registered at the ISD core. Each AS first verifies the signatures, and then computes the fair shares. Fast signing and verification can be implemented using the public-key high-speed signature scheme ed25519. The private key used for signing by an ISD core can be shared among the core ASes in each ISD. For signature verification, the corresponding public key of an ISD core is distributed to all other ISDs, which disseminate the key to all ASes inside the ISD. In practice, on the order of only 100 public keys need to be distributed. Since ISD cores are typically stable, key change and re-distribution should be infrequent. A detailed description is out of scope for this paper.

Dynamic fair sharing. A pertinent question is whether the computed fair share is large enough to be useful. On core links, whose capacity exceeds hundreds of Gbps (recall the example of Australia in Section VIII), even a ratio of only $1 \%$ steady bandwidth is on the order of a few Gbps, which would give each AS outside Australia tens of Mbps of steady traffic.

However, in the destination ISD, given that steady downpaths' capacity is merely a few Mbps of bandwidth, the fair share obtained for each leaf AS is less than $1 \mathrm{kbps}$ - too small to be useful. Of course, a popular destination AS could register several steady down-paths to increase the steady fair share, but the increase factor is still small compared to the number of possible source ASes.

The key observation is that SIBRA enforces the fair share on steady traffic dynamically, i.e., only when a steady downpath becomes congested. The fair share is computed between the ASes that actively use the steady down-path, as opposed to all possible ASes in the Internet. The fair share is reserved for 1 second, which allows the AS two retry attempts if an ephemeral reservation fails (considering an RTT of $300 \mathrm{~ms}-$ a conservative value, according to measurements performed by CAIDA ${ }^{13}$.

\footnotetext{
${ }^{13}$ https://www.caida.org/research/performance/rtt/walrus0202/
} 